\title{
The Joy in imaging the Auger Electron Signal in a FESEM using a Segmented Annular BSED and Stage Bias
}

\author{
B.J. Griffin ${ }^{1}$, A.A. Suvorova ${ }^{1}$, D.C. Joy ${ }^{2}$ and J.R. Michael ${ }^{3}$ \\ 1. Centre for Microscopy, Characterization and Analysis, The University of Western Australia, \\ Crawley, WA Australia. \\ 2. Center for NanoPhase Materials Science, Oak Ridge National Laboratory, Oak Ridge, \\ TN. \\ 3. Sandia National Laboratories, PO Box 5800, Albuquerque, NM.
}

The development and extension of the application of SEM, in all of its variants, over the past 30 years has been inspired by Professor David C. Joy and those he mentored so well. An underlying principle was always that there was more to learn. Professor Joy realized early on that the SEM chamber environment significantly affected the information content of collected images, sometimes in a beneficial way. The following is a recent example.

In the course of investigating the imaging of a freshly-cleaved molybdenum disulphide $\left(\mathrm{MoS}_{2}\right.$ or the naturally occurring 'molybdenite') with a segmented annular BSE detector (DBS) and with stage bias a range of contrasts have been observed and reported ${ }^{1}$. Under specific imaging conditions the carbon-rich support substrate has very high brightness relative to the surrounding MoS2. The initial interpretation, presented orally, was that these images were dominated by the Auger electron signal from the sample. The possibility of Auger electron imaging in a conventional SEM is the focus of this study.

The Auger electron signal from a sample is a well-characterized, low energy component within the BSE range $^{2}$ (fig. 1). It is used widely to image surface detail. The Auger signal is a result of postionisation relaxation of atoms, with a strong, inverse relationship to X-ray yield via a term known as the fluorescent yield (fig. 2). Importantly low $\mathrm{Z}$ elements have high Auger electron yields and the signal is widely employed in EELS and related techniques in TEM. For bulk samples Auger imaging is performed in ultra-high vacuum SEM with specialized energy spectrometers to avoid surface contamination effects.

The experimental configuration and images collected from the MoS2 sample with the various detector elements are shown in fig. 3. The presumed 'Auger-rich' image is presented alone in fig. 4. The sensitivity of the different detector elements to our various conditions of working distance (WD), accelerating voltage (Eo) and stage bias is presented in fig.5, from modelling software generously provided by FEI. An Auger electron spectrum collected from a MoS2 sample, contaminated with a sub-nanometre layer of $\mathrm{C}$ is shown in fig. $6^{3}$. The $\mathrm{C}$ signal is well separated from the Mo L and S K Auger signal by over 100 ev.

The current results of the imaging and modelling are consistent with the various elements acting in concert as a crude energy spectrometer. Given the known energy separation of the $\mathrm{C}$ Auger signal from that of the Mo and $\mathrm{S}$ then an interpretation of fig. 4 as an Auger-dominated signal seem plausible. The stage bias is considered to accelerate the Auger signal from the sample, thereby overcoming conventional barriers to emission in the SEM.

The conclusion is exciting as it offers the potential for the conventional SEM to be additionally used for surface imaging with Auger electrons, under carefully defined conditions. Potential applications include semiconductor QC, materials corrosion and Li-based battery/polymer research. Further studies are in progress and will be reported on [4][5].

References:

[1] Griffin, B.J., Joy, D.C. and Michael, J. Microsc Microanal 20(S2) (2015). 
[2] L. Reimer, Scanning Electron Microscopy (Springer) p. 527.

[3] D.H. Buckley, NASA TN (1970), D-7010.

[4] FEI is acknowledged for access to their DBS modelling software.

[5] Sandia is a multiprogram laboratory operated by Sandia Corporation, a Lockheed Martin Company, for the United States Department of Energy (DOE) under contract DE- AC0494AL85000.

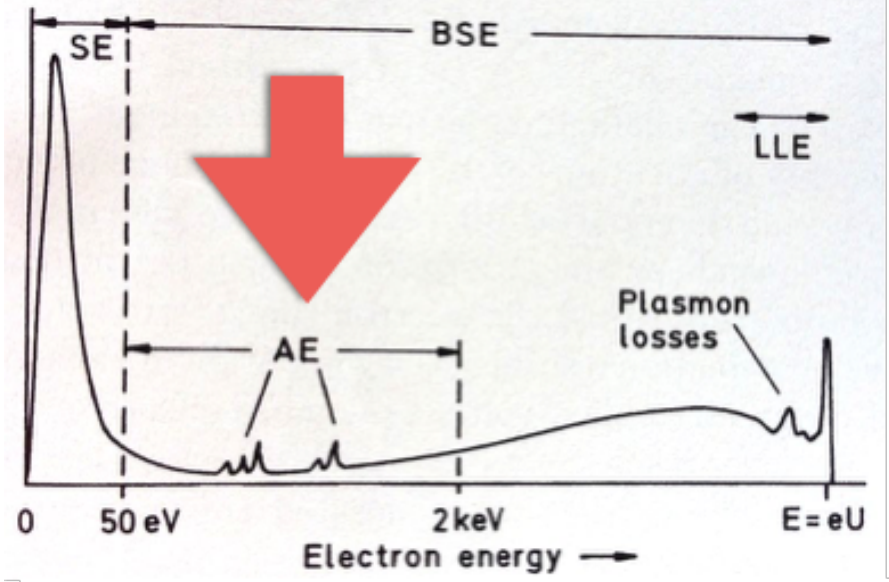

Figure 1: Schematic of the conventional nomenclature of the emitted electron signals with energy (after Reimer, 1998, fig. 1.5).

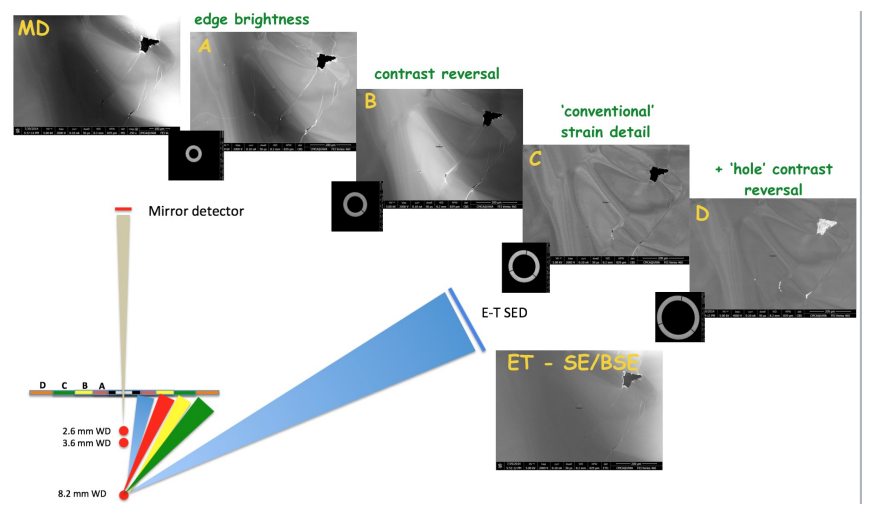

Figure 3: Summary of the experimental configuration and results at $5 \mathrm{kV}, 4 \mathrm{kV}$ stage bias and $8.3 \mathrm{~mm}$ WD.

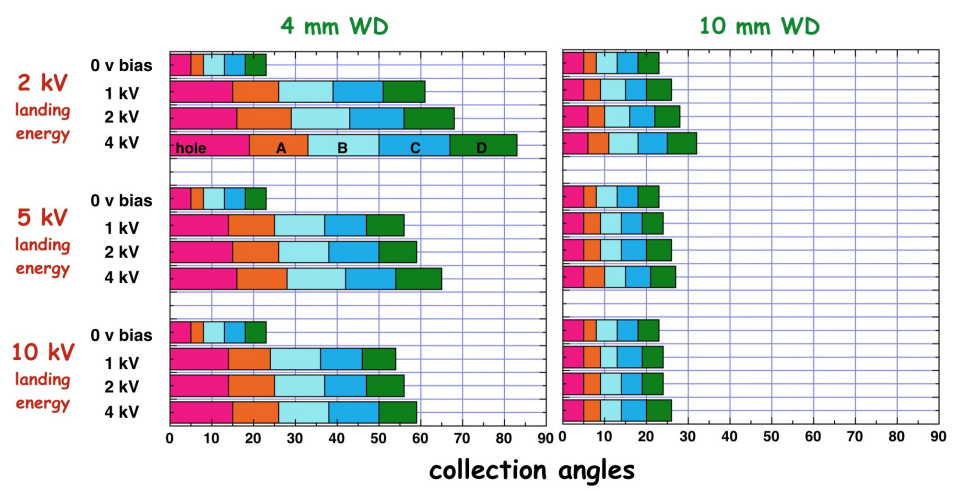

Figure 5: Summary of the modeled DBS ring collection angles as a function of WD, Eo and stage bias.

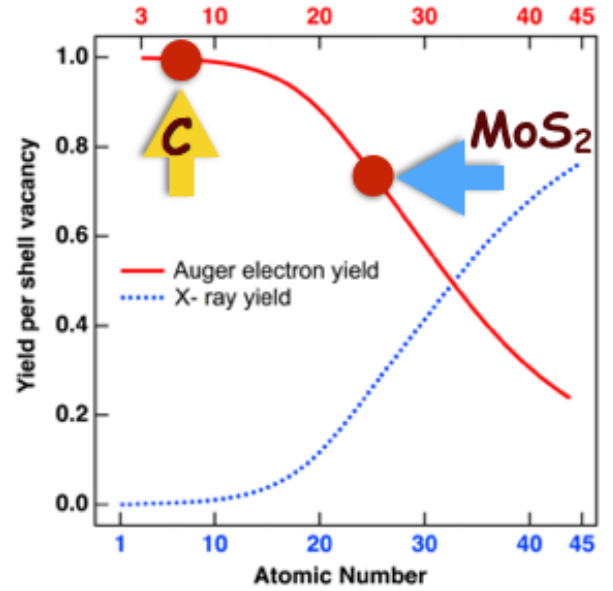

Figure 2: Fluorescence and Auger electron yields as a function of atomic number for $\mathrm{K}$ shell vacancies.

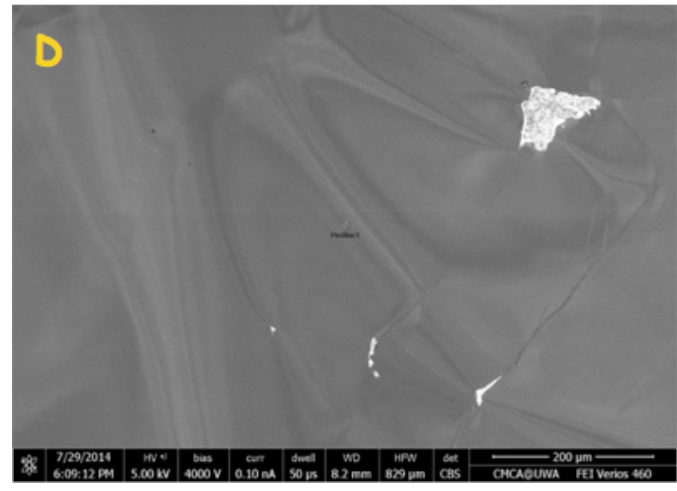

Figure 4: The '(C) Auger electron-rich' BSEI of the MoS2 including exposure of the C-rich substrate at $5 \mathrm{kV}, 4 \mathrm{kV}$ stage bias and the outer ' $\mathrm{D}$ ' BSED ring.

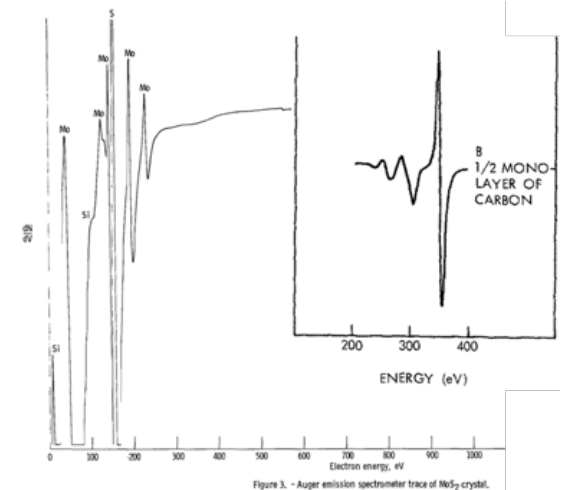

Figure 6: Auger Emission Spectrum of MoS2 with a thin C surface film showing the energy separation of the Auger esignal from the two materials (from Buckley, 1971). 